\title{
Disturbi psichici nella medicina di base e psichiatria territoriale
}

\author{
Psychiatric disorders in primary care and community-based mental health services
}

\author{
GIUSEPPE BERTI CERONI e CECILIA NERI
}

\section{INTRODUZIONE}

Vi sono importanti differenze nel lavoro clinico quotidiano del medico di medicina generale (MMG) e in quello di altri specialisti, ad eccezione forse degli psichiatri che lavorano nei Centri di Salute Mentale territoriali (CSM), che per certi versi si muovono in un ambito simile.

Freeling (1993), uno specialista inglese della medicina di base, mette in luce come caratterizzazioni fondamentali dei MMG il fatto di essere dottori "di primo contatto», di dover tener conto di fattori fisici, psicologici e sociali per poter formulare una diagnosi completa, di necessitare di interventi di ordine igienico ed educativo, oltre che terapeutico, di avere tra i propri scopi la prevenzione ed il controllo della disabilità, oltre che la cura, infine di avere una conoscenza longitudinale del paziente. Tutti questi sono elementi basilari anche nel lavoro degli psichiatri nei CSM.

È però ormai assodato che gli psichiatri vedono solo una minoranza dei pazienti con disturbi psichici, mentre la maggior parte afferiscono agli studi dei MMG e spesso non vengono inviati a consulenza, ma, se riconosciuti, direttamente trattati (Goldberg \& Huxley, 1992). Una recente revisione di Piccinelli (1994) è assai utile per fare il punto degli ormai numerosi studi epidemiologici, al di là delle numerose differenze di metodi.

È altresì dimostrato che, mentre gli psichiatri con-

Indirizzo per la corrispondenza: Prof. G. Berti Ceroni, Servizio di Igiene Mentale e di Assistenza Psichiatrica (SIMAP), Azienda USL della Città di Bologna, c/o Villa Mazzacorati, via Toscana 17/19, 40141 Bologna.

Fax (+39) 051-623.7130. tinuano a vedere per lo più pazienti con disturbi psichici gravi (Olfson et al., 1993), anche nel setting della medicina di base sono presenti «casi» con disturbi relativamente gravi (Berti Ceroni et al., 1992), per lo più affettivi ed ansiosi, anche ad andamento cronico e con alto grado di disabilità, che sono abituali frequentatori di agenzie sanitarie, anche in maniera impropria, e consumatori di numerose indagini diagnostiche (Von Korff et al., 1992).

Infine, studi sulla popolazione o sui pazienti afferenti agli studi dei MMG dimostrano che esistono numerosi soggetti con sintomi depressivi od ansiosi, che tuttavia non soddisfano i criteri di inclusione nei vari disturbi codificati dai diversi sistemi diagnostici, e perciò definiti «casi al limite» o «sotto-soglia» (Gherardi et al., 1991; Goldberg \& Huxley, 1992; Piccinelli et al., 1995); anche questi soggetti sono alti consultatori di servizi sanitari e subiscono menomazioni nella loro vita sociale, come ad esempio perdita di giorni di lavoro (Johnson et al., 1992; Davidson et al., 1994).

Per quanto riguarda la depressione, uno studio recente (Katon et al., 1994) dimostra però che il trattamento specifico (anche farmacologico) da parte di MMG che hanno riconosciuto i propri pazienti come depressi porta a remissione una buona metà dei casi con episodio depressivo maggiore e due terzi dei distimici, dopo solo 4 mesi di trattamento.

Un quadro di tal genere dovrebbe porre ai Centri di Salute Mentale di ogni Dipartimento, e non soltanto ai gruppi specializzati di ricerca, l'esigenza di impostare strategie di comune formazione ed integrazione insieme con i MMG e le loro organizzazioni. Esaminiamo di seguito alcuni studi ed esperienze di rapporto fra psichiatri e MMG che potrebbero dare spunto, in situazioni diverse, a modalità collaborative più formate di quanto abitualmente avviene. 


\section{DALL'EPIDEMIOLOGIA ALLA COLLABORAZIONE NEL RICONOSCIMENTO E NEL TRATTAMENTO}

Gli studi che segnano la storia del rapporto fra medicina di base e psichiatria mostrano difatti uno sviluppo della ricerca nel tempo. Secondo Katon \& Gonzales (1994), si possono raggruppare studi di $1^{\mathrm{a}}, 2^{\mathrm{a}}$ e $3^{\mathrm{a}}$ generazione: i primi riguardano la trasmissione ai MMG di indici di sofferenza psicologica dei vari soggetti (per esempio, alti punteggi a questionari di autovalutazione), per facilitare il riconoscimento di eventuali disturbi; questa procedura si è dimostrata di scarsa utilità. I secondi comportano l'esecuzione di una intervista da parte di uno psichiatra, anche in presenza del MMG, seguita da istruzioni più o meno dettagliate e dall'osservazione nel tempo del decorso clinico; i risultati di queste procedure sono stati anch'essi modesti e discutibili.

L'attenzione riguarda ora soprattutto gli studi di terza generazione, e cioè l'uso da parte dei MMG di interventi più formalizzati. Occorre da un lato continuare ad approfondire la clinica, del tutto peculiare, dei disturbi psichici nella medicina di base, e dall'altro mettere a punto specifiche linee di trattamento. (Saltmore \& Barrowclough, 1993; Üstün \& Gater, 1994).

A nostro avviso paiono promettenti tre direzioni fondamentali: 1) messa a punto di semplici strumenti diagnostici e di precise linee guida di trattamento; 2) iniziative di formazione dei MMG, che forniscano loro un feedback di informazioni su vari pazienti, caso per caso; 3 ) esperienze di integrazione sul campo, che, anche per la medicina di base, mettano alla prova strategie di consulenza e liaison.

\section{Strumenti diagnostici e linee guida di trattamento}

Abbiamo già ricordato che è ben noto, a chi si occupa di disturbi psichici nella medicina di base, che l'utilizzo di sistemi diagnostici con precisi criteri operativi non è del tutto agevole in questo contesto, sia in quanto vi sono situazioni di disagio psicologico, anche importante, che non vengono rilevate da tali strumenti, sia perché la loro articolazione e complessità ne scoraggiano l'uso da parte dei MMG. Per cogliere il disagio psicologico vengono più spesso utilizzati, nella clinica ed anche come screening nella ricerca, questionari auto-valutativi (General Health Questionnaire di Goldberg \& Blackwell, 1970; Symptom
Questionnaire di Kellner, 1987) che misurano il grado di malessere, ma non forniscono una specifica diagnosi, anche se punteggi alti sono fortemente correlati con la presenza di un disturbo diagnosticabile (Rucci et al., 1994).

È perciò di grande interesse la nascita di strumenti specifici che, in un tempo relativamente breve, conducano il MMG ad un orientamento diagnostico più definito. Spitzer et al. (1994) hanno recentemente messo a punto e descritto una scheda diagnostica studiata appositamente per i MMG, il Primary Care Evaluation of Mental Disorders (PRIME - MD), che ha un tempo di compilazione di 8-10 minuti e che permette di diagnosticare con precisione i 5 gruppi di disturbi psichici che più spesso si incontrano nella popolazione generale (disturbi dell'umore, d'ansia, somatoformi, dell'alimentazione e correlati all'alcool). Sappiamo che altri gruppi di ricerca stanno lavorando alacremente in questa direzione.

Per quanto riguarda invece la definizione di linee guida di trattamento specifiche per la medicina generale, ricordiamo in Italia quelle di Bellantuomo et al. (1992) e, per la depressione, le nostre, ormai antiche (Neri et al., 1987); recentemente è stata pubblicata in USA una guideline per la depressione, frutto di una conferenza di consenso nazionale (Depression Guideline Panel, 1993) ed è in corso di pubblicazione quella inerente i disturbi ansiosi. Rush (1993) ha discusso gli innegabili vantaggi che possono sorgere da tale iniziativa, ma anche i possibili danni di una rigida applicazione, volta primariamente al contenimento della spesa.

Per parte nostra, va sempre richiamato il pericolo di costruire linee guida per i MMG esclusivamente da parte di psichiatri, senza avvalersi della esperienza dei medici stessi; questo assunto, che da tempo aprioristicamente avevamo stabilito, è stato potentemente confermato nel corso della collaborazione, ormai quadriennale, che abbiamo con $30 \mathrm{MMG}$ identificati dall'Ordine dei Medici e della Società Italiana di Medicina Generale di Bologna per far parte del Progetto Collaborativo "Bologna - Psichiatria e Medicina di Base» con psichiatri delle Aziende USL e della Clinica Psichiatrica I. All'interno del progetto è stato fra l'altro possibile esaminare con precisione il pattern prescrittivo di codesti MMG (Berti Ceroni et al., 1995).

\section{Interventi di formazione dei Medici di Medicina Generale}

Goldberg (1992) propone di suddividere i pazienti 


\section{G. Berti Ceroni e C. Neri}

con disagio psicologico afferenti agli ambulatori medici in tre gruppi: situazioni che hanno bisogno di riconoscimento e discussione, casi che richiedono un intervento sociale, pazienti che necessitano di un più specifico intervento medico e psicologico.

Per quanto riguarda il riconoscimento dei pazienti che necessitano di cure, i fenomeni tipici che rendono molti casi non riconosciuti sono una presentazione «somatica» piuttosto che "psicologica» del problema, la scarsa evidenza della sofferenza psicologica da parte del paziente e, da parte del medico, l'incapacità di dar risposta alle comunicazioni verbali e non verbali del paziente riguardo ai suoi problemi emotivi (Goldberg, 1990). L'autore suggerisce di cominciare l'intervista con domande aperte, via via poi dirette sempre più strettamente sul problema focale.

Per consentire l'acquisizione di un'adeguata capacità comunicativa, Gask \& McGrath (1989) suggeriscono di sostituire al tradizionale rapporto di consulenza-consulto un metodo in cui il MMG in prima persona possa utilizzare gli strumenti dell'attenzione agli aspetti emozionali e dell'empatia, spartendoli con lo psichiatra. Tale capacità può essere sviluppata attraverso il percorso formativo di cui Lesser (1985) in Canada e Gask et al. (1987) a Manchester forniscono una dettagliata dimostrazione. Dal «colloquio orientato al problema» di Lesser, Goldberg, Gask ed i loro collaboratori hanno sviluppato una tecnica di discussione in gruppo, fra MMG e psichiatri, di videotapes su colloqui clinici. Alla fine del training, che consta di numerose occasioni di discussione a cadenza ravvicinata, viene valutata la capacità acquisita da ogni MMG ad opera di un supervisore «in cieco", che cioè non sa se il colloquio è stato registrato prima o dopo gli incontri.

Per quanto riguarda lo specifico problema della presentazione somatica del disturbo emotivo, la tecnica più pertinente pare quella della «riattribuzione del sintomo", che propone l'utilizzazione di un atteggiamento empatico e di attenzione ai disturbi riferiti, fino al punto di riuscire a stabilire il collegamento, nel corso del colloquio, fra disturbo somatico e sofferenza psicologica (Goldberg et al., 1989).

L'importanza di queste esperienze formative predisposte dagli studiosi canadesi ed inglesi sta nella possibilità, per i MMG, di acquisire uno strumento di intervento, che fa riferimento ad un modello «standardizzato» e che è quindi ripetibile anche in altre esperienze.

A Bologna, nell'ambito del Progetto Collaborativo succitato, stiamo sperimentando la validità di un progetto di formazione per i $M M G$, ispirato alle esperienze degli autori anglosassoni. Nel frattempo è in via di conclusione la valutazione di una strategia di intervento fondata sulla discussione in gruppo, fra uno psichiatra esperto e alcuni (3-5) MMG, dei casi identificati mediante una procedura a due stadi negli studi di codesti medici; il risultato di questa procedura viene valutato, mediante un follow-up ad un anno, rispetto all'andamento dei casi di un gruppo di controllo di MMG, che li hanno gestiti direttamente, anche tramite invio a consulenza secondo le normali procedure previste dalla convenzione per i MMG.

\section{L'interazione pratica tra medici e psichiatri}

Seppure con molto ritardo rispetto allo sviluppo della psichiatria di consulenza e liaison negli ambiti ospedalieri, di cui hanno recentemente effettuato una revisione critica Mayou (1994) e Balestrieri (1994), vi è un crescente interesse nel mondo anglosassone per l'integrazione dell'attività di MMG e psichiatri negli ambulatori. Questa metodica di lavoro, applicata in particolare ai disturbi psicologici propri della medicina di base, permette di migliorare il rapporto costo-efficacia degli interventi.

Tyrer e i suoi collaboratori (Tyrer, 1984; Tyrer et al., 1984) furono i primi a descrivere l'esperienza di psichiatri al lavoro in ambulatori dei MMG di Nottingham, in confronto con la attività svolta presso l'ospedale psichiatrico: l'accesso risultò più gradito ai pazienti, che consideravano privo di stigma tale invio, tant'è che molti $(19 \%)$ affermavamo che non si sarebbero recati negli ambulatori dell'ospedale psichiatrico. Questa esperienza si è in seguito ampliata (Ferguson et al., 1992), rendendo molto più agevole l'uso di varie strutture sanitarie multidisciplinari, fra cui i day hospitals, e permettendo un miglior monitoraggio longitudinale dei pazienti.

L'utilizzazione di figure non mediche (infermieri ed assistenti sociali) fa sì che gli psichiatri, da unici dispensatori di cure, divengano coordinatori e valutatori di attività svolte insieme da MMG e da altre figure professionali (Schuster et al., 1994). Jackson et al. (1993) descrivono un servizio ambulatoriale multidisciplinare, che si avvale di psicologi, infermieri psichiatrici, assistente sociale e di due psichiatri a tempo parziale, in diretta connessione con i MMG; nel loro studio, che confronta consulenza tradizionale ed invio all'équipe multidisciplinare, gli autori notano che il lavoro di équipe permette di aumentare il numero di casi valutati, raggiungendo nel primo an- 
no di attività più del doppio rispetto agli utenti dei consulenti tradizionali negli ambulatori dell'ospedale psichiatrico; inoltre solo i casi gravi accettavano di andare in consulenza all'ospedale psichiatrico, con un tasso di drop out molto maggiore (quasi 5 volte).

In Italia, dove così esteso è stato l'impianto di servizi psichiatrici territoriali con èquipes multidisciplinari, vi è tuttavia scarsità di notizie di modalità collaborative specifiche fra MMG e psichiatri, che curino l'integrazione piuttosto che regolare semplicemente l'invio. Le sole eccezioni, a nostra conoscenza, sono l'«ambulatorio di pronta consulenza» costituito a Reggio Emilia per l'accoglienza e la gestione di pazienti inviati dai MMG per disturbi lievi o moderati (Asioli et al., 1995) e la «consulenza telefonica» in via di attivazione a Bologna, dove psichiatri esperti sono disponibili per una consulenza, anche ripetuta, a quei medici che si trovano alle prese con casi problematici, nei quali non è ancora proponibile l'invio del paziente alla consulenza (Paltrinieri et al., 1995).

\section{CONCLUSIONI}

È dubbio se si può già affermare che sta nascendo una psichiatria di consulenza e liaison anche nei confronti della medicina di base, per lo meno al livello raggiunto in ospedale generale, che pur solleva tante riserve (Balestrieri, 1994): da un lato infatti abbiano l'evidenza di un fiorire di studi relativi all'allestimento di strumenti diagnostici semplici ed efficaci, di tecniche formative robustamente codificate, di attività lavorative integrate; dall'altro resta il sospetto che queste iniziative siano concentrate in poche aree territoriali e in pochi gruppi di ricerca e che non abbiano ancora peso nelle effettive modalità di prestazione. Sorprende, in particolare, che così poche siano le iniziative ben formate e riconoscibili in Italia, dove generalizzata è la presenza dei MMG ed estesa e consistente quella di servizi psichiatrici territoriali; pesa forse ancora la principale ragion d'essere, anti-manicomiale, della riforma psichiatrica, che porta molti psichiatri ad occuparsi intensivamente dei casi più gravi e di considerare scarsamente rilevante l'impegno riguardo a disturbi più moderati.

Una considerazione finale è, nella nostra esperien$z a$, indispensabile: programmi e procedure di formazione e di integrazione debbono nascere dalla inizia- tiva congiunta di psichiatri e di MMG. Per ora, a Bologna, la spinta, anche molto forte e continua, non si è canalizzata attraverso i Servizi di Medicina di Base delle USL o attraverso la Regione, ma attraverso la Società Italiana di Medicina Generale che, anche a livello nazionale, pare molto interessata allo sviluppo di iniziative che diano ai MMG risorse effettive per fronteggiare i problemi posti da una buona quota dei loro pazienti.

\section{BIBLIOGRAFIA}

Asioli F., Bologna M., Contini G. \& Jonus A. (1995). Il circuito medicina di base e psichiatria: lo studio e l'organizzazione a Reggio Emilia. Rivista Sperimentale di Freniatria (in stampa).

Balestrieri M. (1994). Psichiatria di consulenza e liaison. Cosa bolle in pentola? Epidemiologia e Psichiatria Sociale 3, 139143.

Bellantuomo C., Balestrieri M., Ruggeri M. \& Tansella M. (1992). I Disturbi Psichici nella Medicina Generale. Il Pensiero Scientifico Editore: Roma.

Berti Ceroni G., Berti Ceroni F., Bivi R., Corsino M.A.,De Marco P., Gallo E., Giovannini G., Gherardi S., Pezzoli A., Rucci P. \& Neri C. (1992). DSM-III mental disorders in general medical sector: a follow up and incidence study over a two year period. Social Psychiatry and Psychiatric Epidemiology 27, 234-241.

Berti Ceroni G., Berardi D., Monari M. \& Pezzoli A. (1995). Il trattamento farmacologico dei disturbi psichici da parte dei medici di medicina generale. Rivista Sperimentale di Freniatria (in stampa).

Davidson J.R., Hughes D.C., George L.K. \& Blazer D.G. (1994). The buondary of social phobia. Exploring the threshold. Archives of General Psychiatry 51, 975-983.

Depression Guideline Panel (1993). Depression in Primary Care: Detection, Diagnosis and Treatment. Clinical Practice Guideline: Quick Reference Guide for Clinicians. US Dept. of Health and Human Services, Agency for Health Care Policy and Research: Rockville, Md.

Ferguson B., Cooper S., Brothwell J., Markantonakis A. \& Tyrer P. (1992). The clinical evaluation of a new community psychiatric service based on general practice psychiatric clinics. British Journal of Psychiatry 160, 493-497.

Freeling P. (1993). Diagnosis and treatment of depression in general practice. British Journal of Psychiatry 163, 14-19.

Gask L. \& Mc Grath C. (1989). Psychotherapy and general practice. British Journal of Psychiatry 154, 445-453.

Gask L., Mc Grath C., Goldberg D. \& Millar T. (1987). Improving the psychiatric skills of established general practitioners: evaluation of group teaching. Medical Education 21, 362-368.

Gherardi S., Berti Ceroni F., Bivi R., Corsino M.A., De Marco P., Neri C., Pezzoli A. \& Rucci P. (1991). Sofferenza psichica e diagnosi psichiatrica. il problema operativo dell'identificazione del «caso» nei contesti sanitari di base. In Psichiatria $e$ Medicina di Base (ed. F. Asioli F. e G. Contini). Clueb: Bologna.

Goldberg D. (1990) Reasons for misdiagnosis. In Psychological Disorders in General Medical Settings (ed. N. Sartorius, D. 
Goldberg, G. de Girolamo, J.A. Costa e Silva, Y. Lecrubier and H.U. Wittchen). Hogrefe \& Huber Publishers: Toronto.

Goldberg D. (1992). A classification of psychological distress for use in primary care settings. Social Science and Medicine 35,189-193.

Goldberg D. \& Blackwell B. (1970). Psychiatric illness in general practice. A detailed study using a new method of case identification. British Medical Journal 2, 439-443.

Goldberg D. \& Huxley P. (1992). Common Mental Disorders. A Bio-social Model. Tavistock: London.

Goldberg D., Gask L. \& O'Dwod T. (1989). The treatment of somatisation: teaching techniques of reattribution. Journal of Psychosomatic Research 33, 689-695.

Jackson C., Gater R., Goldberg D., Tantam D., Loftus L. \& Taylor H. (1993). A new community mental health team based in primary care. British Journal of Psychiatry 162, 375-384.

Johnson J., Weissmann M.H. \& Klerman G. (1992). Service utilisation and social morbidity associated with depressive symptoms in the community. Journal of American Medical Association 18, 1478-1483.

Katon W. \& Gonzales J. (1994). A review of randomized trials of psychiatric consultation liaison studies in primary care. Psychosomatics 35, 268-278.

Katon W., Lin E., Von Korff M., Bush T., Walker E., Simon C. \& Robinson P. (1994). The predictors of persistence of depression in primary care. Journal of Affective Disorders 31, 81-90.

Kellner R. (1987). A symptom questionnaire. Journal of Clinical Psychiatry 48, 268-274.

Lesser A.L. (1985). Problem based interviewing in general practice: a model. Medical Education 19, 299-304.

Mayou R. (1994). Clinical significance of psychiatric disorder in the general hospital. Epidemiologia e Psichiatria Sociale 3, 145-150.

Neri C., Berti Ceroni F., Corsino M.A. \& Pezzoli A. (1987). La depressione vista in contesti non psichiatrici: una rassegna. Rivista di Psichiatria 22, 312-325.

Olfson M., Klerman G. \& Pincus H.A. (1993). The role of psychiatrists in organized outpatient mental health settings. American Journal of Psychiatry 150, 4, 625-631.
Paltrinieri E., Berti Ceroni G., Gherardi S. \& Neri C. (1995). La consulenza telefonica nella medicina di base. Rivista Sperimentale di Freniatria (in stampa).

Piccinelli M. (1994). Disturbi psichici nella medicina generale: perché tante differenze? Epidemiologia e Psichiatria Sociale 3, 195-208.

Piccinelli M., Pini S., Bellantuono C., Bonizzato P., Paltrinieri E., Ustun T.B., Sartorinius N. \& Tansella M. (1995). Lo studio internazionale multicentrico dell'Organizzazione Mondiale della Sanità sui disturbi psichici nella medicina generale: risultati relativi all'area di Verona. Epidemiologia e Psichiatria Sociale 4, 27-50.

Rucci P., De Marco P. \& Bivi R. (1994). Il Symptom Questionnaire come strumento di screening nei contesti sanitari di base. Epidemiologia e Psichiatria Sociale 3, 31-38.

Rush A.J. (1993). Clinical practice guidelines. Archives of General Psychiatry 50, 483-490.

Saltmore S. \& Barrowclough C. (1993). Mental health and primary care. Current Opinion in Psychiatry 6, 258-262.

Schuster J.M., Kern E.E., Kane V. \& Nettleman L. (1994). Changing roles of mental health clinicians in multidisciplinary teams. Hospital and Community Psychiatry 45, 1187-1189.

Spitzer R., Williams J., Kroenke R., Linzer M., Verloin de Gruy F., Hahn S., Broody D. \& Johnson J.G. (1994). Utility of a New Procedure for diagnosing mental disorders in primary care, Journal of American Medical Association 272, 1749-1756.

Tyrer P. (1984). Psychiatric clinics in general practice. An extension of community care. British Journal of Psychiatry 145, 914.

Tyrer P., Seivewright N. \& Wollerton S. (1984). General practice psychiatric clinics. Impact on psychiatric services. British Journal of Psychiatry 145, 15-19.

Üstün T.B. \& Gater R. (1994). Integrating mental health into primary care. Current Opinion in Psychiatry 7, 173-180.

Von Korff M., Ormel J., Katon W. \& Lin E. (1992). Disability and depression among high utilizers of health care. Archives of General Psychiatry 49, 91-100. 